\title{
A low-complexity QoS aware resource allocation algorithm for multi pair device-to-device (D2D) communications
}

\author{
Hamidreza Bagheri $^{1}\left[\right.$ | Fernando Alberto Miranda Bonomi ${ }^{2} \mid$ Marcos Katz $^{1}$
}

${ }^{1}$ Centre for Wireless Communications, University of Oulu, Oulu, Finland

${ }^{2}$ Laboratorio de Telecomunicaciones, DEEC FACET, Universidad Nacional de Tucumán, San Miguel de Tucumán, Argentina

\section{Correspondence}

Hamidreza Bagheri, Centre for Wireless Communications, University of Oulu, Oulu, Finland.

Email: hamidreza.bagheri@ee.oulu.fi

\begin{abstract}
Device-to-device (D2D) communication underlaying cellular networks is considered a promising technology to enhance network throughput, spectral efficiency, and performance of next generation networks. However, these potential gains hinge on the exploiting mechanism for resource sharing between cellular users (CUs) and D2D pairs. In this paper, we analytically formulate the problem of resource sharing as an optimization problem to maximize network throughput while guaranteeing the required quality-of-service (QoS) for both cellular and D2D users. We propose a low-complexity four-step resource allocation algorithm to address the optimization problem. We exploit a distance-based method to derive a resource reuse candidacy graph (RCG) and three exclusive regions (ERs) to evaluate the suitability of resource sharing between each CU and D2D pair. Then, we use a paring mechanism to find the optimal set of D2D pairs for spectrum sharing with each CU to maximize network throughput. The performance of the proposed algorithm is investigated in terms of network throughput, outage probability, and computational complexity. Numerical results show that the proposed algorithm provides high throughput and spectrum utilization with low complexity while efficiently guaranteeing QoS for CUs and D2D pairs.
\end{abstract}

\section{1 | INTRODUCTION}

The increasing penetration of smart handheld devices coupled with the widespread use of bandwidth-hungry applications such as video streaming and multimedia content sharing have generated a drastic increase in mobile data traffic. This massive growth in traffic volume already pushed the limits of the current wireless technologies particularly from the spectrum resources standpoint and opened up a new field of research for next generation $(5 \mathrm{G})$ wireless communication technology. The recently introduced concept of spectrum sharing-based device-to-device (D2D) communications is being touted as a promising enabling technology for $5 \mathrm{G}$ networks. D2D communications improve quality-of-service (QoS) of users and network performance, as well as utilization in operators' radio resources. ${ }^{1-4}$ Although D2D communications offer plenty of benefits, as the result of spectrum sharing between cellular and local communications, there are a number of technical challenges that need to be tackled. Resource allocation and interference management are considered as the two most critical technical challenges for this technology. In fact, mutual interference between cellular and D2D communications can significantly decrease the network performance. Therefore, an efficient resource-sharing algorithm and interference coordination mechanism must be formulated to achieve higher spectrum utilization and guarantee target performance levels for both cellular and D2D users. Fodor et $\mathrm{al}^{5}$ have introduced the concept of network-assisted D2D communications and discussed the general technical challenges of D2D communications such as peer and service discovery, physical layer design, mode selection, power control, and interference management. D2D 
communications underlay of a cellular network are studied as an efficient mechanism to reduce traffic on licensed cellular network in Doppler et al. ${ }^{6}$ Huang et $\mathrm{al}^{7}$ and Hakola et $\mathrm{al}^{8}$ have proposed the concept of mode selection to determine the optimum transmission mode and spectrum sharing for D2D communications. $\mathrm{Xu}$ et $\mathrm{al}^{9}$ have adopted exclusion regions and open-loop fraction power control method to mitigate the D2D to cellular user (CU) interference in multicell uplink spectrum-sharing scenarios. Lin et $\mathrm{al}^{10}$ and Lee et $\mathrm{al}^{11}$ propose power control mechanisms to manage the mutual interference between cellular and D2D communications. To mitigate the interference from CUs to D2D communication links, a distance-constrained uplink resource-sharing method is proposed in Wang and Chu. ${ }^{12}$ An interference-limited area (ILA) control scheme has been proposed in Min et $\mathrm{al}^{13}$ to manage the interference between cellular and D2D communications. By driving the coverage of ILA, a CU is selected to share its uplink resources with a D2D pair that can lead to system capacity improvement. Golrezaei et a ${ }^{14,15}$ have introduced an attractive concept for utilization. The idea is to exploit D2D communications and distributed caching network for content delivery among mobile devices. In Bagheri et al ${ }^{16}$ we exploit the concepts of full-duplex D2D communications to improve the throughput and spectral efficiency of the network. To date, numerous resource allocation algorithms have been developed for concurrent communications of multiple D2D links. Zulhasnine et al ${ }^{17}$ proposed a greedy heuristic algorithm to address a mixed integer nonlinear programming resource allocation problem, and Kaufman et $\mathrm{al}^{18}$ proposed a fixed power margin scheme to manage the mutual interference between cellular and D2D pairs. In $\mathrm{Ni}$ et al, ${ }^{19}$ a sector-based geometrical method is applied for uplink resource allocation and to improve network throughput with multiple D2D communications. In Bagheri and Katz, ${ }^{20}$ we proposed an efficient mechanism to coordinate the intracell interference and enhance the overall system sum-rate when a group of D2D pairs communicate by reusing the resources of a $\mathrm{CU}$. Xu et $\mathrm{al}^{21}$ and $\mathrm{Li}$ et $\mathrm{al}^{22}$ addressed the D2D resource allocation using reverse combinatorial auction (R-ICA) mechanisms. Authors have considered spectrum resources as a set of resource units auctioned off by groups of D2D pairs in sequence. Kebriaei et $\mathrm{al}^{23}$ Song et $\mathrm{al}^{24}{ }^{24}$ and $\mathrm{Li}$ et $\mathrm{al}^{25}$ consider the D2D communication from a game-theoretic approach. Research work in previous studies ${ }^{26-28}$ have studied joint power control and resource allocation for multiple D2D pairs communication. Joint mode selection and power control mechanisms have been developed in $\mathrm{Yu}$ et $\mathrm{al}^{29}$ and Jung et $\mathrm{al}^{30}$ to address the resource allocation problem and improve the overall throughput for D2D communications underlaying cellular networks.

To the best of our knowledge, the vast majority of the existing work has restricted the spectrum sharing between CUs and only one D2D pair to prevent co-channel interference among different D2D pairs. In fact, this limitation results in being unable to exploit the actual potential of resource sharing for spectrum utilization. However, some recent research has tackled the problem of resource allocation for multi-D2D link aiming at maximizing network throughput. The main problem with the recent proposed algorithms is that they cannot maintain QoS for cellular and D2D communications. From another perspective, a great deal of research has focused on power control mechanisms to reduce the transmitting interference to CUs and D2D pairs. One can speculate that the interference level does not depend only on transmit power but also the use of an effective resource allocation mechanism can reduce the interference level on users. Besides, although related studies are rich in addressing interference management between cellular and D2D communications, they mainly concentrate on uplink resources. Moreover, they suffer from high computational complexity.

The objective of this paper is to effectively address the simultaneous communications of multiple D2D pair by sharing spectrum resources of each CU with multiple D2D pairs and providing high throughput and spectrum efficiency while guaranteeing QoS for both cellular and D2D communications. We analytically model and formulate the problem of resource allocation for D2D pairs as an optimization problem to maximize network throughput and guarantee the required QoS for CUs and D2D pairs. Furthermore, we derive a closed-form expression for the outage probabilities of CUs when it shares its spectrum resources with multiple D2D pairs. To attain the aforementioned goals, we propose a novel and low-complexity QoS aware heuristic resource allocation algorithm (QARA), which is constructed in four consecutive steps. In Step 1, we adapt a QoS aware admission control mechanism by developing a weighted resource reuse candidacy graph (RCG) to determine which D2D pairs can reuse spectrum resources of a $\mathrm{CU}$ without violating the predefined QoS requirement for CUs. Step 2 describes a mechanism to choose an optimal D2D pair for paring with each active CU to maximize the achievable throughput. In Step 3, we derive and use three exclusive regions (ERs) to investigate the candidacy and admission control of multiple D2D pairs for resource sharing for each active CU. Step 4 investigates an optimal pairing strategy for finding the optimal set of D2D pairs for resource sharing with each active $\mathrm{CU}$ to maximize the overall network throughput. To evaluate the proposed algorithm, we compare performance of QARA algorithm with R-ICA,${ }^{21}$ the Hungarian algorithm, ${ }^{31,32}$ and conventional random resource allocation in terms of network throughput, outage probability, and computational complexity. The derived results show that QARA provides near optimal network throughput with low computational complexity. Moreover, QARA can efficiently guarantee QoS for CUs and D2D pairs and also significantly improves spectrum utilization. 
The remainder of the paper is organized as follows. Section 2 describes the system model of D2D communications underlaying cellular network. In Section 3, the optimization problem is analytically formulated. Section 4 outlines the proposed resource allocation algorithm. Section 5 demonstrates the numerical results and discusses the performance of the proposed algorithm. Finally, conclusions and further discussions are given in Section 6.

\section{2 | SYSTEM MODEL DESCRIPTION}

In this paper, we consider downlink resource sharing in an isolated cell, where $N$ CUs represented by $C=\left\{c_{1}, c_{2}, \ldots, c_{N}\right\}$ and $M$ D2D pairs denoted by $D=\left\{d_{1}, d_{2}, \ldots, d_{M}\right\}$ are distributed all over the cell and spectrum resources of a $\mathrm{CU}$ can be shared with one or multiple D2D pairs, as illustrated in Figure 1. Each D2D link is made up of a D2D transmitter (D2D-Tx) and a D2D receiver (D2D-Rx). We investigate the spatial distribution of CUs and D2D pairs to determine which CU and D2D pair(s) can share the same spectrum resources without violating the QoS constraints defined for CUs and D2D pairs. The key idea for throughput maximization is to minimize the mutual interference between cellular and D2D communications generated due to spectrum sharing. As we will discuss in Section 4, the expected outcome of QARA algorithm will be a set of disjoint resource-sharing groups (RSGs) showing D2D pairs that are admitted to reuse spectrum resources of each particular CU. The RSGs are denoted by $G_{i}=\left\{d_{k}\right\}$, where $G_{i} \subseteq D, \bigcup_{i=1}^{N} G_{i}=D, i \in\{1,2, \ldots, N\}$, and $k \in\{1,2, \ldots, k\}$. For instance in Figure $1, G_{1}=\left\{d_{1}, d_{2}\right\}$ means that D2D pairs $d_{1}$ and $d_{2}$ are admitted to reuse spectrum resources of the $\mathrm{CU} c_{1}$. The other resource-sharing groups in this example are $G_{2}=\left\{d_{3}\right\}, G_{3}=\left\{d_{4}\right\}$, and $G_{4}=\left\{d_{5}, d_{6}\right\}$.

In this paper, we are primarily interested in a loaded D2D network where the number of D2D pairs is larger than CUs. An application of this scenario is a cooperative content delivery where users can exchange their content on D2D links. We have discussed D2D communications technologies in Bagheri et al. ${ }^{4}$ The network throughput is measured by the system sum-rate, and QoS is measured by the signal-to-interference-plus-noise ratio (SINR) on CUs and D2D-Rxs. We would like to distinguish between active and other CUs. We define a CU as active if it can share its resources with D2D pair(s) without violation of its QoS requirement.

We assume that the cellular communications takes place underlay orthogonal frequency division multiple access (OFDMA) downlink system and under control of the serving base station (BS). As each CU is allocated with independent orthogonal frequencies, there will be no interference between CUs in the cell. It is assumed that the BS is the principal

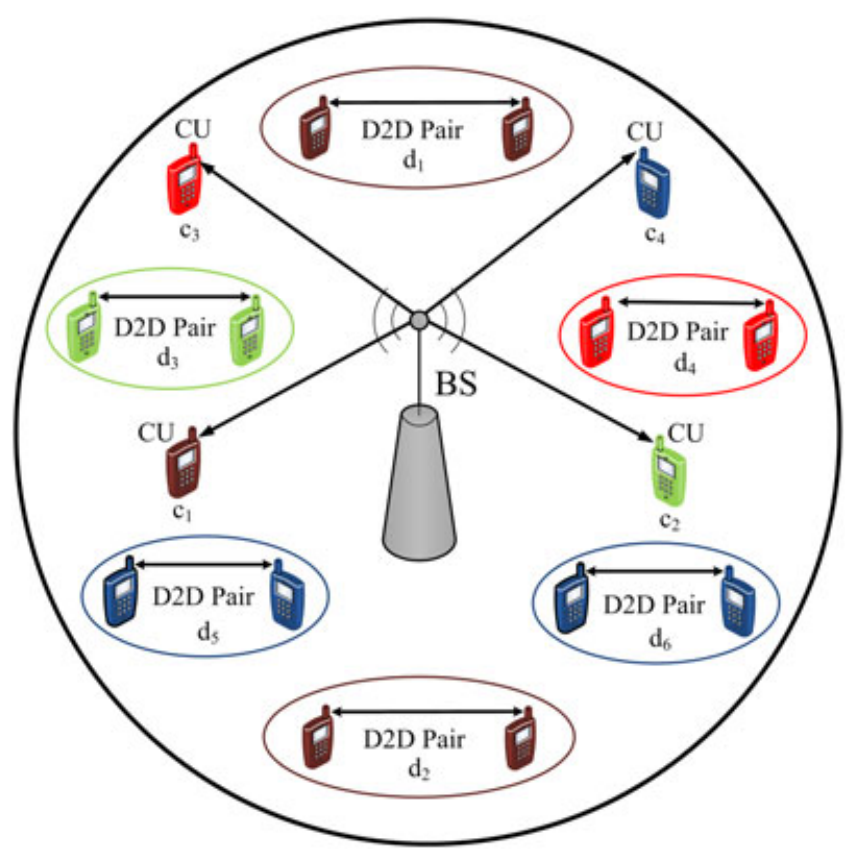

FIGURE 1 A system model of resource sharing between CUs and D2D pairs.

decision maker for resource allocation, and it is perfectly aware of channel state information of all the involved links. We use a simple transmit power adaption method ${ }^{30}$ on the BS for cellular communications.

\section{Interference Problem Formulation}

When a CU shares its allocated downlink resources with a D2D pair, the CU is exposed to interference by the D2D-Tx, while the D2D-Rx is victim of severe interference from the BS. Accordingly, when a CU and several D2D pairs communicate on shared spectrum of the CU simultaneously, interference management becomes more complex, and one needs to deal with 3 types of intracell interference as follows: (1) interference from D2D-Txs to the CU, (2) interference from the BS to D2D-Rxs, and (3) interference from D2D-Tx of each pair to D2D-Rxs of other pairs in a same RSG. Note that in a more realistic scenario, intercell interference should also be taken into account. All these interference types have to be limited to obtain the predefined QoS for the CU and D2D pairs. To get a better insight into the problem, let us consider a scenario in which D2D-Tx 1 and D2D-Tx 2 transmit by reusing the downlink spectrum resources of $c_{1}$ while the BS transmit to $c_{1}$, as illustrated in Figure 2. Since the BS and D2D-Txs transmit at the same time on a shared medium, the BS creates severe interference to both D2D-Rxs, and each D2D-Tx transmits interference to the $\mathrm{CU} c_{1}$ as well as the other pair's D2D-Rx.

Let us assume that spectrum resources allocated to the $i$-th $\mathrm{CU}$ is shared with $K$ D2D pairs in $G_{i}=\left\{d_{k}\right\}$, where $k \in$ $\{1,2, \ldots, k\}$. During the downlink period, the BS transmits data $x_{i}^{c}$ to the $i$-th $\mathrm{CU}$, and $K$ D2D-Txs transmit their data 


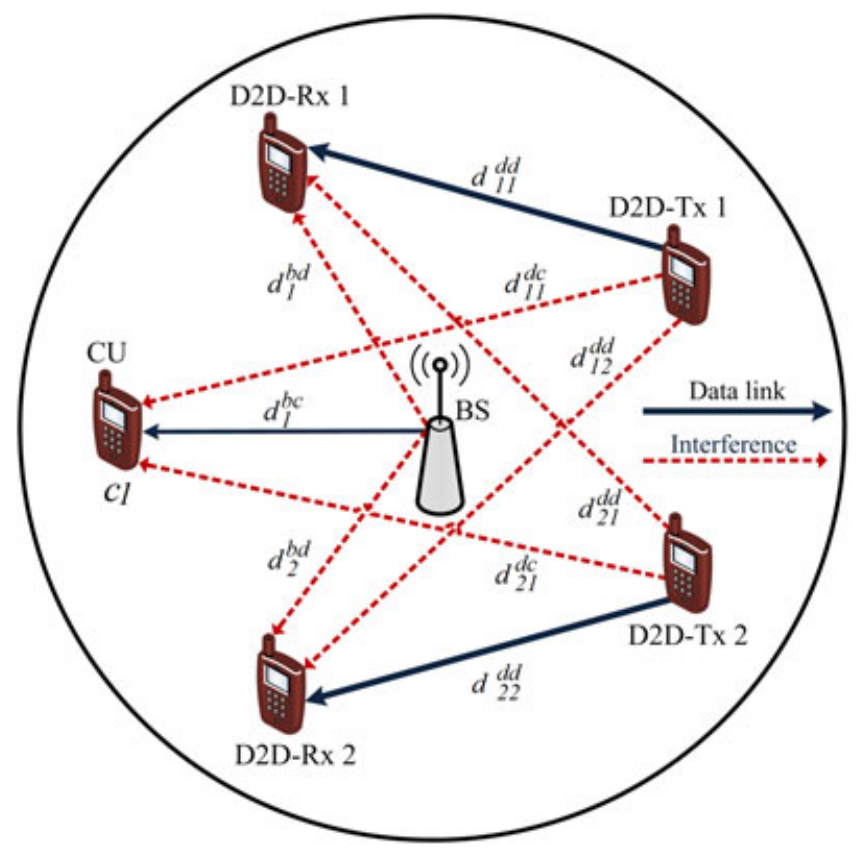

FIGURE 2 Interference problem when a CU and two D2D pairs communicate on shared downlink resources.

content such as $x_{k}^{d}$ to their corresponding D2D-Rxs at the same time, using the same spectrum resource. The received signal at the $i$-th $\mathrm{CU}, y_{i}^{c}$, and the $k$-th D2D-Rx, $y_{k}^{d}$, can be written as

$$
\begin{gathered}
y_{i}^{c}=\sqrt{P_{i}^{b c}\left(d_{i}^{b c}\right)^{-\alpha}} h_{i}^{b c} x_{i}^{c} \\
+\sum_{d_{k} \in G_{i}} \sqrt{P_{k}^{d}\left(d_{k i}^{d c}\right)^{-\alpha}} h_{k i}^{d c} x_{k}^{d}+P_{n} . \\
y_{k}^{d}=\sqrt{P_{k}^{d}\left(d_{k k}^{d d}\right)^{-\alpha}} h_{k k}^{d d} x_{k}^{d}+\sqrt{P_{k}^{b d}\left(d_{k}^{b d}\right)^{-\alpha}} h_{k}^{b d} x_{i}^{c} \\
+\sum_{d_{l} \in G_{i}-\left\{d_{k}\right\}} \sqrt{P_{l}^{d}\left(d_{l k}^{d d}\right)^{-\alpha}} h_{l k}^{d d} x_{l}^{d}+P_{n},
\end{gathered}
$$

where $P_{i}^{b c}$ and $P_{k}^{b d}$ are the transmit power of the BS to the $i$-th $\mathrm{CU}$ and $k$-th D2D-Rx; $P_{k}^{d}$ and $P_{l}^{d}$ are transmit power of $k$-th and $l$-th D2D-Txs; $P_{n}$ denotes the power spectral density of additive white Gaussian noise. $\alpha$ stands for path-loss exponent. $h_{i}^{b c}, h_{k}^{b d}, h_{k i}^{d c}, h_{k k}^{d d}$, and $h_{l k}^{d d}$ represent channel coefficients of the links between the BS to the $i$-th $\mathrm{CU}$ and $k$-th D2D-Rx, $k$-th D2D-Tx to the $i$-th $\mathrm{CU}$, corresponding D2D-Rx of the same pair, and D2D-Tx of a pair to D2D-Rxs of the other pairs, respectively. We assume Rayleigh fading channels, and for simplicity, $\mathbb{E}\left|h_{i}^{b c}\right|^{2}=1, \mathbb{E}\left|h_{k}^{b d}\right|^{2}=1, \mathbb{E}\left|h_{k i}^{d c}\right|^{2}=1$, $\mathbb{E}\left|h_{k k}^{d d}\right|^{2}=1, \mathbb{E}\left|h_{l k}^{d d}\right|^{2}=1 . d_{i}^{b c}$, and $d_{k}^{b d}$ are the distances of the BS to the $i$-th CU and $k$-th D2D-Rx; $d_{k i}^{d c}$ and $d_{k k}^{d d}$ denote the distances of the $k$-th D2D-Tx to the $i$-th CU and D2D-Rx of the same pair, while $d_{l k}^{d d}$ denotes the distance from l-th D2D-Tx to $k$-th D2D-Rx. Note that $G_{i}-\left\{d_{k}\right\}$ denotes all D2D pairs in $G_{i}$ except $k$-th $\mathrm{D} 2 \mathrm{D}$ pair.

\section{3 | OPTIMIZATION PROBLEM FORMULATION}

In this section, we analytically model and formulate the problem of resource allocation for D2D pairs as an optimization problem to maximize network throughput while guaranteeing the QoS for CUs and D2D pairs. Finally, we derive and discuss the outage probability of the sharing $\mathrm{CU}$ analytically. We aim to maximize network throughput when a CU shares its spectrum resources with multiple D2D pairs. We argue that increasing the number of D2D pairs admitted to reuse spectrum resources of each $\mathrm{CU}$ can increase spectrum utilization as well as network throughput, if the mutual interference between the $\mathrm{CU}$ and admitted $\mathrm{D} 2 \mathrm{D}$ pairs is managed effectively. Assuming sharing of resources of the $i$-th CU with $K$ admitted D2D pairs in $G_{i}$, throughput can be computed by sum-rate of traditional cellular link and all D2D pairs. Accordingly, the achievable throughput can be written as $R_{i}^{c}+\sum_{d_{k} \in G_{i}} R_{k}^{d}$, where using Shannon theorem $R_{i}^{c}=$ $\log _{2}\left(1+\gamma_{i}^{c}\right)$ and $R_{k}^{d}=\log _{2}\left(1+\gamma_{k}^{d}\right)$ are the achievable throughput for the $i$-th CU and $k$-th D2D pair, respectively. $\gamma_{i}^{c}$ and $\gamma_{k}^{d}$ represent the SINR at the $i$-th $\mathrm{CU}$ and $k$-th D2D pair, respectively, and can be written as

$$
\begin{gathered}
\gamma_{i}^{c}=\mathbb{E}\left(\frac{P_{i}^{b c}\left|h_{i}^{b c}\right|^{2}\left(d_{i}^{b c}\right)^{-\alpha}}{\sum_{d_{k} \in G_{i}} P_{k}^{d}\left|h_{k i}^{d c}\right|^{2}\left(d_{k i}^{d c}\right)^{-\alpha}+P_{n}}\right), \\
=\frac{P_{i}^{b c}\left(d_{i}^{b c}\right)^{-\alpha}}{\sum_{d_{k} \in G_{i}} P_{k}^{d}\left(d_{k i}^{d c}\right)^{-\alpha}+P_{n}} . \\
\gamma_{k}^{d}=\mathbb{E}\left(P_{k}^{d}\left|h_{k k}^{d d}\right|^{2}\left(d_{k k}^{d d}\right)^{-\alpha} /\left(P_{k}^{b d}\left|h_{k}^{b d}\right|^{2}\left(d_{k}^{b d}\right)^{-\alpha}\right.\right. \\
\left.\left.+\sum_{d_{l} \in G_{i}-\left\{d_{k}\right\}} P_{l}^{d}\left|h_{l k}^{d d}\right|^{2}\left(d_{l k}^{d d}\right)^{-\alpha}+P_{n}\right)\right) \\
P_{k}^{d}\left(d_{k k}^{d d}\right)^{-\alpha} \\
P_{k}^{b d}\left(d_{k}^{b d}\right)^{-\alpha}+\sum_{d_{l} \in G_{i}-\left\{d_{k}\right\}} P_{l}^{d}\left(d_{l k}^{d d}\right)^{-\alpha}+P_{n}
\end{gathered}
$$

where $\sum_{d_{k} \in G_{i}} P_{k}^{d}\left(d_{k i}^{d c}\right)^{-\alpha}$ represents the sum of transmitting interference from all the D2D pairs in $G_{i}$ to the $i$-th $\mathrm{CU}$ and $\sum_{d_{l} \in G_{i}-\left\{d_{k}\right\}} P_{l}^{d}\left(d_{l k}^{d d}\right)^{-\alpha}$ represents the sum of transmitting interference of D2D-Txs in $G_{i}$ to the $k$-th D2D-Rx.

To maximize the overall network throughput where $N$ CUs share their resources with $M$ D2D pairs, we formulate the overall network throughput maximization problem as

$$
\begin{gathered}
\max \left\{\sum_{c_{i} \in C} R_{i}^{c}+\sum_{d_{j} \in D} \sum_{c_{i} \in C} \beta_{i j}^{c d} R_{j}^{d}\right\} \\
\text { s.t. } \mathrm{C} 1: \quad \gamma_{i}^{c} \geq \gamma_{\text {min }}^{c}, \\
\mathrm{C} 2: \quad \gamma_{j}^{d} \geq \gamma_{\text {min }}^{d},
\end{gathered}
$$


where $\gamma_{\min }^{c}$ and $\gamma_{\min }^{d}$ denote the minimum required SINR on each CU and D2D-Rx, respectively. $\beta_{i j}^{c d}$ is a binary variable as the resource reuse indicator in which $\beta_{i j}^{c d}=1$ when $\mathrm{D} 2 \mathrm{D}$ pair $d_{j}$ is admitted to reuse spectrum resources of $c_{i}$, otherwise $\beta_{i j}^{c d}=0$. In addition, we need to ensure that each D2D pair is assigned with spectrum resources of one of the active CUs. The constraints $\mathrm{C} 1$ and $\mathrm{C} 2$ represent the QoS requirements for $\mathrm{CU}$ and $\mathrm{D} 2 \mathrm{D}$ pairs, respectively, and imply that to achieve the maximum throughput, the instantaneous SINR of each CU and D2D pairs in its resource-sharing group should be higher or equal than the predetermined SINR targets.

It can be seen that the maximization problem formulated in Equation 5 is a kind of NP-hard combinatorial optimization problem. It is generally difficult to obtain a globally optimal solution for such a problem. Hence, the solution for Equation 5 can be mapped to a heuristic algorithm to find the best set of paired CUs and D2D pairs that maximize network throughput and satisfy the constraints of the problem. The key issue of the solution is to exploit an efficient mechanism to investigate which CU and D2D pairs can be properly paired up to maximize the overall throughput while guaranteeing the required QoS at each CU and D2D-Rx. Next, on the basis of the constraints of the optimization problem, we investigate the outage probability of the sharing CUs.

\section{Outage Probability}

Interference management is the key challenge for resource sharing and throughput improvement in D2D communications. As discussed earlier, with downlink resources sharing between a CU and multiple D2D pairs, the CU and D2D-Rxs are exposed to interference, and hence, increasing the number of D2D pairs will increase the level of interference. Accordingly, increasing in the number of sharing D2D pairs can decrease the SINR of the CU dramatically, if no effective mechanism for resource sharing is implemented. Thus, we introduce Definition 1 to consider outage probability of a CU when it shares its spectrum resources with multiple D2D pairs.

Definition 1. We define the outage probability of the $C U c_{i}$ as the probability that its instantaneous SINR falls below a predetermined threshold denoted by $\gamma_{\min }^{c}$

$$
P_{i}^{\text {Out }, c}=\operatorname{Pr}\left(\frac{P_{i}^{b c}\left|h_{i}^{b c}\right|^{2}\left(d_{i}^{b c}\right)^{-\alpha}}{\sum_{d_{k} \in G_{i}} P_{k}^{d}\left|h_{k i}^{d c}\right|^{2}\left(d_{k i}^{d c}\right)^{-\alpha}+P_{n}} \leq \gamma_{\min }^{c}\right) .
$$

We introduce Lemma 1 to compute the outage probability of the $i$-th CU.

Lemma 1. If we assume $Z_{i}^{b c}=P_{i}^{b c}\left|h_{i}^{b c}\right|^{2}\left(d_{i}^{b c}\right)^{-\alpha}$ and $Z_{k i}^{d c}=$ $P_{k}^{d}\left|h_{k i}^{d c}\right|^{2}\left(d_{k i}^{d k}\right)^{-\alpha}$ are independent exponentially distributed random variables with mean values $\bar{Z}_{i}^{b c}$ and $\bar{Z}_{k i}^{d c}$, respectively, then the outage probability in Equation 8 can be rewritten as

$$
P_{i}^{\text {Out }, c}=\operatorname{Pr}\left(\frac{Z_{i}^{b c}}{\sum_{d_{k} \in G_{i}} Z_{k i}^{d c}+P_{n}}\right) .
$$

The outage probability of the $i$-th $C U$ can be written in a closed-form expression as

$$
P_{i}^{\text {Out }, c}=1-e^{-\frac{\gamma_{\min }^{c} P_{n}}{\bar{Z}_{i}^{b c}}} \prod_{d_{k} \in G_{i}}\left(\frac{1}{1+\gamma_{\min }^{c} \overline{\bar{Z}}_{k i}^{d c}}\right) .
$$

The proof of Lemma 1 is presented in the Appendix.

\section{4 | QUALITY-OF-SERVICE AWARE HEURISTIC RESOURCE ALLOCATION ALGORITHM}

The QARA algorithm is conducted in 4 steps that are summarized in Table 1. It is obvious that network throughput and spectrum utilization is highly dependent on efficiency of the algorithm for interference mitigation and number of concurrent D2D communications. Before diving into detail explanation of the algorithm, let us introduce some concepts involved in the proposed algorithm.

Definition 2. A resource reuse candidacy graph $(R C G)$ is $a$ weighted bipartite graph $G=\left(V_{C}, V_{D}, E_{R}\right)$ that has 2 sets of vertexes of active $C U s$ and $D 2 D$ pairs represented by $C=\left\{c_{1}, c_{2}, \ldots, c_{N}\right\}$ and $D=\left\{d_{1}, d_{2}, \ldots, d_{M}\right\}$, respectively. Each edge of graph shows the achievable throughput with pairing $a C U$ and $a D 2 D$ pair.

TABLE 1 Main steps of the QARA algorithm

Step 1: Quality-of-service aware admission control for single D2D pairs. For each active CU, find a list of candidate D2D pairs that can share the same spectrum without violating constraint $\mathrm{C} 1$ of the problem.

Step 2: Paring strategy for a single D2D pair. For each active CU, consider all available candidate D2D pairs and choose the pair that maximizes throughput.

Step 3: Quality-of-service aware admission control for multiple D2D pairs. Determine D2D pairs that can join a RSG without violating QoS constraints.

Step 4: Resource allocation for multiple D2D pairs. For each RSG, choose the D2D pair that maximizes throughput (if any).

Steps 3 and 4 are applied iteratively until no more pairing is possible. 


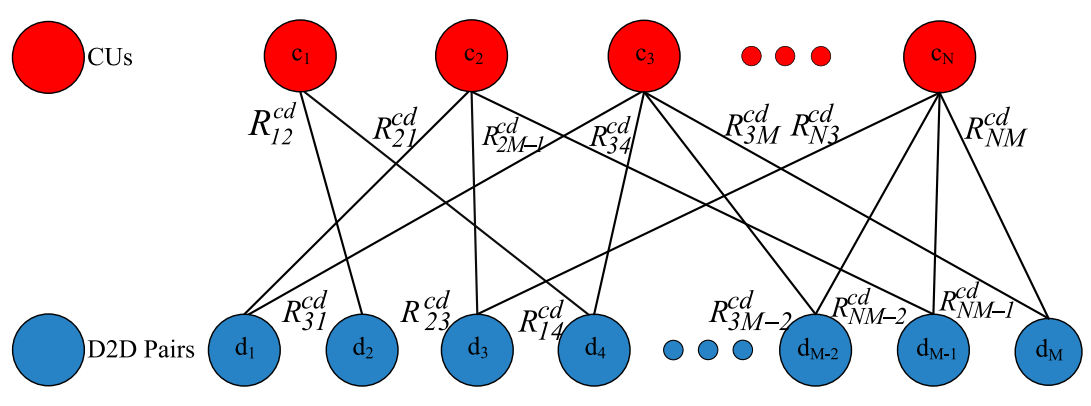

FIGURE 3 An example of RCG (reuse candidacy graph).

Definition 3. For each active $C U$, using $R C G$, we derive a set of admissible D2D pairs showing by $C_{i}=\left\{d_{j}\right\}$ where $i \in\{1,2, \ldots, N\}$ and $j \in\{1,2, \ldots, M\}$.

\section{Step 1: Quality-of-service aware admission control for D2D pairs}

In this step, we construct and exploit an RCG to investigate which CU and D2D pair(s) can communicate on shared spectrum without violating the required QoS at the CU. The RCG can be seen as an admission control algorithm to indicate whether $d_{j}$ is admitted to reuse spectrum resources of $c_{i}$ or not. Before discussing the RCG construction, we introduce proposition 1 to consider the criterion that determines which D2D pair can reuse spectrum resource of a CU.

Proposition 1. The D2D pair $d_{j}$ is a candidate to reuse spectrum resources of the $C U c_{i}$ if $d_{j i}^{d c} \geq d_{\mathrm{min}, i}^{d c}$, where $d_{\mathrm{min}, i}^{d c}$ is the minimum separation distance of D2D pairs to the $C U c_{i}$ to guarantee its required $Q o S$ and given by

$$
d_{\min , i}^{d c}=\left(\frac{P_{j}^{d} \gamma_{\min }^{c}}{P_{i}^{b c}\left(d_{i}^{b c}\right)^{-\alpha}-\gamma_{\min }^{c} P_{n}}\right)^{\frac{1}{\alpha}} .
$$

It is straightforward to derive Equation 11 using constraint (Equation 6). Proposition 1 implies that $d_{j}$ is admissible for resource sharing with $c_{i}$ if the actual distance between $c_{i}$ and the D2D-Tx of $d_{j}$ is larger than $d_{\min , i}^{d c}$ In other words, a CU and a D2D pair can be paired up only if the D2D pair does not cause QoS violation at the CU. To construct the RCG and with respect to proposition 1 , we use an association indicator $c_{i j}$ as

$$
c_{i j}=\left\{\begin{array}{l}
1, \text { if proposition } 1 \text { is satisfied } \\
0, \text { otherwise }
\end{array}\right.
$$

$c_{i j}=1$ implies that there is an edge between $c_{i}$ and $d_{j}$ in RCG, and $c_{i j}=0$ signifies $c_{i}$ and $d_{j}$ do not communicate on shared spectrum. Using RCG and for each CU, we can obtain the D2D pair candidates to reuse spectrum resources of each $\mathrm{CU}$ $c_{i}$ denoted by $C_{i}=\left\{d_{j} \mid c_{i j}=1\right\} . C_{i}=\emptyset$ implies that $c_{i}$ does not share its resources with any D2D pair. On the basis of the constructed RCG, an edge of the graph is established between a CU and D2D pair if Equation 12 is satisfied. Hence, if an edge between $c_{i}$ and $d_{j}$ is established, the weight of the edge can be computed by

$$
R_{i j}^{c d}=R_{i}^{c}+R_{j}^{d},
$$

where $R_{i j}^{c d}$ is the total achievable throughput when $c_{i}$ and a single D2D pair $d_{j}$ communicate on shared spectrum, $R_{i}^{c}$ and $R_{j}^{d}$ are achievable throughput of CU $c_{i}$ and D2D pair $d_{j}$ when they share the same spectrum resources. It should be noted that different distribution of CUs and D2D pairs may lead to different RCG formation. An example of RCG is presented in Figure 3.

\section{Step 2: Paring strategy for a single D2D pair}

In this step, based on the constructed RCG, for each CU, we consider which D2D pair in its candidate list provides higher throughput if it will be paired up with that CU. The D2D pair maximizing the total throughput is called the optimal D2D pair and can be chosen by

$$
d_{j^{*}}=\underset{d_{j}}{\operatorname{argmax}}\left(R_{i j}^{c d}\right),
$$

where $j^{*}$ is the index of the admitted D2D pair to be added to $G_{i}$. This means that for each CU $c_{i}$, we consider all D2D pairs in its candidates list $C_{i}$ and choose D2D pair with the highest $R_{i j}^{c d}$. If two D2D pairs have the same values for $R_{i j}^{c d}$, we choose the one that has larger distance to that CU. By this step, we obtain the optimal solution of Equation 5 for a single D2D pair and $G_{i}$ for all active CUs has exactly one member.

\section{Step 3: Quality-of-service aware admission control for multiple D2D pairs}

When a new D2D pair accesses to spectrum of a CU that already has shared its spectrum resources with some other D2D pairs in $G_{i}$, because of the shared medium, it may generate interference to the CU and D2D-Rx(s) in the $G_{i}$. We argue that for each active $\mathrm{CU}$, there should be a minimum separation distance between D2D-Txs and the CU that guarantees the required QoS for the CU. Moreover, to ensure the QoS for a D2D-Rx, there should exist a minimum separation distance between the D2D-Rx and the BS and interfering D2D-Txs. In this step, we derive the above mentioned minimum separation distances, and based on these geometric parameters, we derive three circular ERs to determine the admissible D2D 
pairs for resource sharing with each active CU. Here, we introduce proposition 2 to determine the $\mathrm{D} 2 \mathrm{D}$ pairs that can reuse spectrum resources of a $\mathrm{CU}$.

Proposition 2. For each $C U c_{i}$, a D2D pair $d_{j}$ in $C_{i}$ is admissible to reuse spectrum resources of the $C U c_{i}$, which already shared its spectrum with at least one D2D pair in $G_{i}$, if it is not located in $E R_{d c}, E R_{b d}$, and $E R_{d d}$. Next, we discuss how to derive the ERs.

Defining $\mathrm{ER}_{\mathrm{dc}}$

Considering the same transmit power for all D2D-Txs, the interference level at CUs is mainly dependent on the distance of D2D-Txs to the CU sharing the spectrum. In fact, to guarantee the required QoS for a $\mathrm{CU}$, there should exist a minimum separation distance from the interfering D2D-Txs. According to constraint $\mathrm{C} 1$ in Equation 6,

$$
\frac{P_{i}^{b c}\left(d_{i}^{b c}\right)^{-\alpha}}{\sum_{d_{k} \in G_{i}} P_{k}^{d}\left(d_{k i}^{d c}\right)^{-\alpha}+P_{n}} \geq \gamma_{\min }^{c}
$$

We derive an $\mathrm{ER}_{\mathrm{dc}}$ for each $\mathrm{CU}$ with radius of $r_{i}^{d c}$ that can be computed as

$$
r_{i}^{d c}=\left(\frac{\sum_{d_{k} \in G_{i}} P_{k}^{d} \gamma_{\min }^{c}}{P_{i}^{b c}\left(d_{i}^{b c}\right)^{-\alpha}-\gamma_{\min }^{c} P_{n}}\right)^{\frac{1}{\alpha}}
$$

\section{Defining $\mathrm{ER}_{\mathrm{bd}}$}

The level of transmitting interference to a D2D-Rx is dependent on its distance from the BS and interfering D2D-Txs. To ensure the QoS on a D2D-Rx, there should be a minimum separation distance of the D2D-Rx from the BS and interfering D2D-Txs that can limit the aggregated interference at the D2D-Rx. According to constraint C2 in Equation 7,

$$
\frac{P_{k}^{d}\left(d_{k k}^{d d}\right)^{-\alpha}}{P_{k}^{b d}\left(d_{k}^{b d}\right)^{-\alpha}+\sum_{d_{l} \in G_{i}-\left\{d_{k}\right\}} P_{l}^{d}\left(d_{l k}^{d d}\right)^{-\alpha}+P_{n}} \geq \gamma_{\min }^{d} .
$$

We derive a circular $\mathrm{ER}_{\mathrm{bd}}$ with the radius of $r_{k}^{b d}$ as

$r_{k}^{b d}=\left(P_{k}^{b d} \gamma_{\min }^{d} /\left(P_{k}^{d}\left(d_{k k}^{d d}\right)-\gamma_{\min }^{d}\left(\sum_{d_{l} \in G_{i}-\left\{d_{k}\right\}} P_{l}^{d}\left(d_{l k}^{d d}\right)^{-\alpha}+P_{n}\right)\right)\right)^{\frac{1}{\alpha}}$.

\section{Defining $\mathrm{ER}_{\mathrm{dd}}$}

To mitigate harmful interference of D2D-Txs to D2D-Rxs of other pairs, we apply the similar process in $\mathrm{ER}_{\mathrm{bd}}$ to obtain the radius of the $\mathrm{ER}_{\mathrm{dd}}$ as

$$
r_{k}^{d d}=\left(\frac{\gamma_{\min }^{d} \sum_{d_{l} \in G_{i}-\left\{d_{k}\right\}} P_{l}^{d}}{P_{k}^{d}\left(d_{k k}^{d d}\right)^{-\alpha}-\gamma_{\min }^{d}\left(P_{k}^{d}\left(d_{k k}^{d d}\right)^{-\alpha}+P_{n}\right)}\right)^{\frac{1}{\alpha}}
$$

Equation 19 implies that multiple D2D pairs can communicate on shared spectrum with $k$-th $\mathrm{D} 2 \mathrm{D}$ pair, if none of their D2D-Txs is located in the ER of the $k$-th $\mathrm{D} 2 \mathrm{D}$ pair.

\section{Step 4: Optimal resource allocation for multiple D2D pairs}

In this step, we investigate an optimal paring strategy to improve the overall network throughput. For resource sharing with each active CU, we need to find the optimal set of D2D pairs that can maximize network throughput without violating its QoS requirement. The optimal D2D pair for resource sharing with $\mathrm{CU} c_{i}$, which has already been paired up with at least one D2D pair in the first two steps, can be found by

$$
d_{j^{*}}=\underset{d_{j}}{\operatorname{argmax}}\left(R_{i k}^{c d}+R_{j}^{d}\right)
$$

where $R_{i k}^{c d}=R_{i}^{c}+\sum_{d_{k} \in G_{i}} R_{k}^{d}$ is the sum of the achievable throughput by each CU $c_{i}$ and $K$ admitted D2D pair(s) in $G_{i}$. The QARA algorithm is shown in Figure 4. With respect to QARA algorithm, the complexity of Step 1 and Step 2 for pairing at most one $\mathrm{D} 2 \mathrm{D}$ pair per active $\mathrm{CU}$ is upper

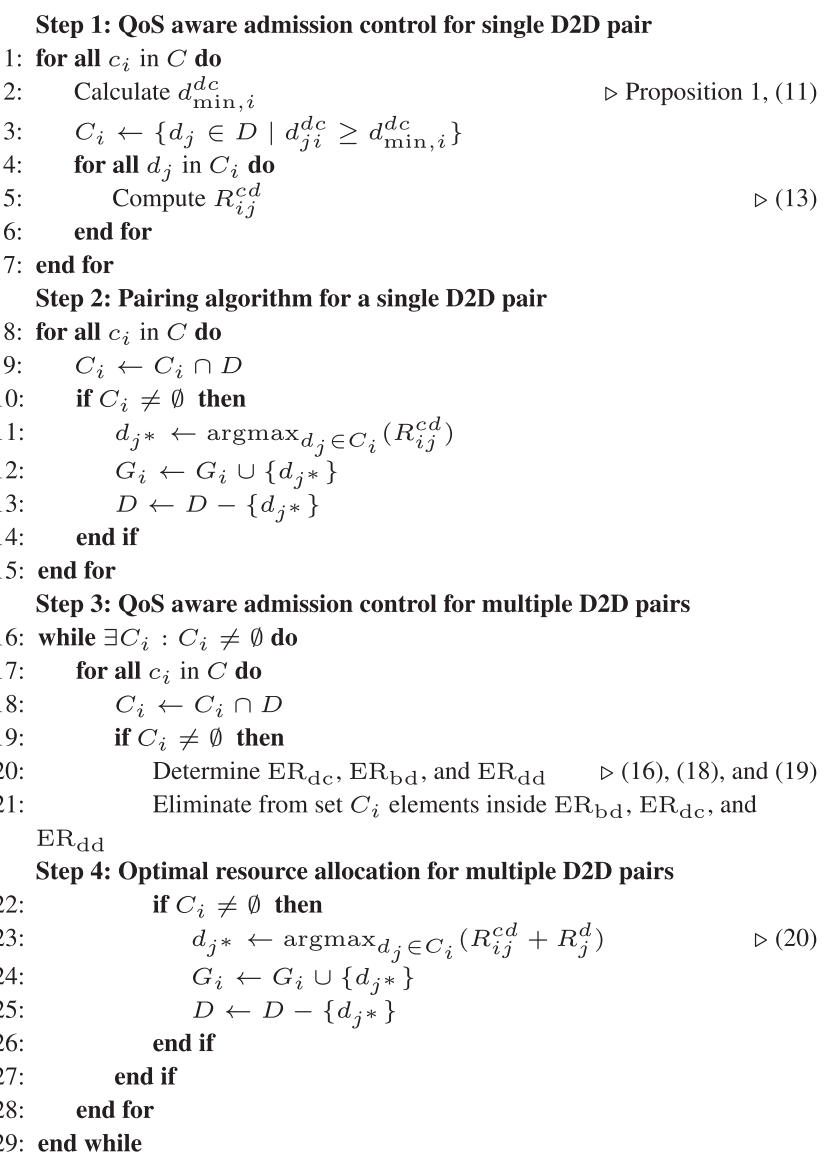

FIGURE 4 QARA (Quality-of-service aware heuristic resource allocation) algorithm. 
bounded by $O(N M)$. The outer loop of Steps 3 and 4 for sharing spectrum resources with multiple D2D pairs runs $O(N)$ times and inner loop runs $O\left(M^{2}\right)$ in the worst case. Thus, complexity of QARA algorithm in the worst case is $O\left(N M^{3}\right)$.

\section{5 | NUMERICAL RESULTS AND DISCUSSIONS}

In this section, we perform extensive simulation experiments to evaluate the proposed algorithm through Monte Carlo simulations in MATLAB. We consider a single circular cell with a radius of $500 \mathrm{~m}$, where the BS is located in the center of cell and CUs and D2D pairs are uniformly distributed all over the cell. The maximum distance between the D2D-Tx and D2D-Rx of a D2D pair is restricted between 10 to $50 \mathrm{~m}$. To investigate the proposed algorithm, we compare the performance of QARA algorithm with R-ICA, the Hungarian algorithm (HA), and random resource allocation (RA) in terms of network throughput, outage probability, and computational complexity. To control the interference from the BS to D2D-Rxs, we exploit an adaptive transmit power for the BS and fixed transmit power for D2D-Txs in our simulations for all the considered algorithms. The common parameters $^{29,30}$ used for simulation experiments are summarized in Table 2.

\section{1 | Geometric constraints}

We begin our analysis by discussing the minimum separation distances required to ensure the predefined QoS at CUs and D2D pairs. Then, to get a better insight on the impact of the QoS requirements and distances on the number of admissible D2D pairs, we consider the ERs establishment in six different configurations.

TABLE 2 Simulation parameters

\begin{tabular}{|ll}
\hline Parameter & \multicolumn{1}{c}{ Value } \\
\hline System bandwidth & $5 \mathrm{MHz}$ \\
\hline BS max transmission power & $30 \mathrm{dBm}$ \\
\hline UE max transmission power & $23 \mathrm{dBm}$ \\
\hline Noise spectral density & $-174 \mathrm{dBm} / \mathrm{Hz}$ \\
\hline Path-loss model for cellular links & $128.1+37.6 \log (d[\mathrm{~km}])$ \\
\hline Path-loss model for D2D links & $148+40 \log (d[\mathrm{~km}])$ \\
\hline QoS requirement for CUs & $10 \mathrm{~dB}$ \\
\hline QoS requirement for D2DU-Rxs & $5 \mathrm{~dB}$ \\
\hline Number of CUs & 8 \\
\hline Number of D2D pairs & $2-16$ \\
\hline
\end{tabular}

\subsection{1 | Minimum separation distances}

Figure 5 shows the minimum separation distance between D2D-Tx(s) and the CU to guarantee $10 \mathrm{~dB}$ SINR at the CU when it shares its resources with 1 to $10 \mathrm{D} 2 \mathrm{D}$ pairs and its distance to the BS varies between 1 and $500 \mathrm{~m}$. As can be observed, the required minimum distances increase with increasing the distance of CU to the BS and number of admitted D2D pairs. As we used adaptive transmission power on the $\mathrm{BS}$, for CUs located more than $300 \mathrm{~m}$ away from the BS, the $\mathrm{BS}$ has to transmit with its maximum transmission power. To guarantee the required QoS at the CU when it shares the spectrum with 3 D2D pairs and distance between the BS and the CU is less than $300 \mathrm{~m}$, D2D-Txs need to be $150 \mathrm{~m}$ away from the CU. But for the same situation when the distance between the BS and the CU is $450 \mathrm{~m}$, D2D-Txs need to be kept 300 to $350 \mathrm{~m}$ away from the CU. In addition, when CU is located $450 \mathrm{~m}$ away from the BS (near to the cell edge) and more than 8 D2D pairs share the same spectrum, D2D-Txs need be 400 to $450 \mathrm{~m}$ away from the CU. Note that for lower values of the required QoS at the $\mathrm{CU}$, the minimum separation distance of D2D-Txs to the CU decreases.

Figure 6 depicts the minimum separation distance of D2D-Rxs from the BS for $\gamma_{\text {min }}^{c}=10 \mathrm{~dB}$ and $\gamma_{\text {min }}^{d}=5 \mathrm{~dB}$. It can be seen that the minimum required distance from the $\mathrm{BS}$ to D2D-Rx increases with increasing D2D link length (i.e.,

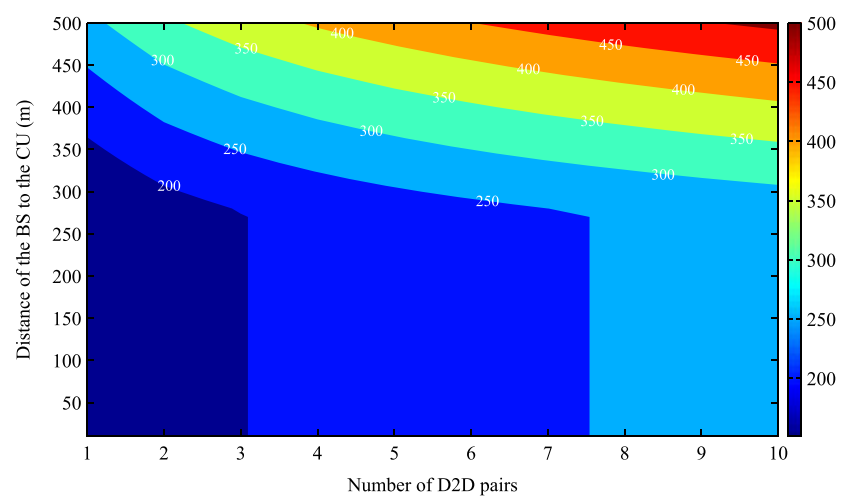

FIGURE 5 Minimum separation distance of D2D-Txs to the CU.

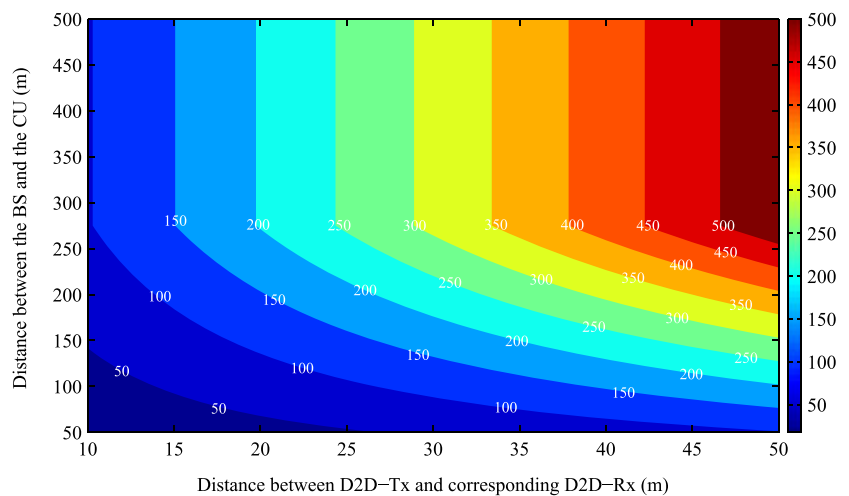

FIGURE 6 Minimum separation distance of D2D-Rxs from the BS. 


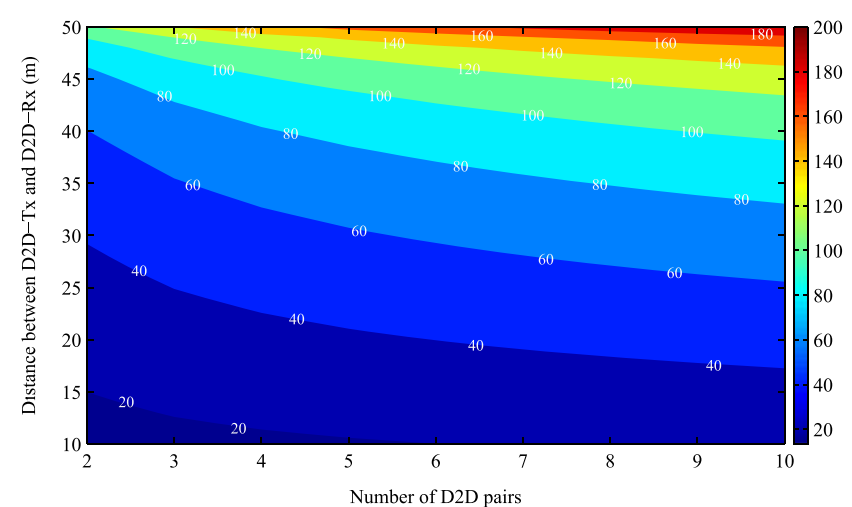

FIGURE 7 Minimum separation distance of D2D-Rx from D2D-Txs of other pairs

distance between D2D-Tx and it corresponding D2D-Rx) and distance of CU from the BS. However, D2D link length shows greater impact on the required separation distance. For instance, when a CU is $300 \mathrm{~m}$ away from the BS and D2D link length is less than $15 \mathrm{~m}$, the required QoS for D2D-Rx can be guaranteed with less than $150 \mathrm{~m}$ distance of D2D-Rx from the BS. However, when D2D link length is $50 \mathrm{~m}$, to guarantee QoS at D2D-Rx, it needs to be kept $500 \mathrm{~m}$ away from the BS.

Figure 7 illustrates the minimum separation distance of D2D-Rx from D2D-Txs of other pairs when the distance between transmitter and receiver of a D2D pair is between 10 to $50 \mathrm{~m}$ and the number of D2D pairs is between 2 and 10 . The required QoS for a D2D-Rx is set to $5 \mathrm{~dB}$. Because of the fixed and low transmission power of D2D-Txs, the minimum separation distance of D2D-Rx from interfering D2D-Txs grows with increasing in D2D links length. It also grows as the number of D2D pairs sharing the same spectrum resources increases, due to the greater number of interferers. When there are only $2 \mathrm{D} 2 \mathrm{D}$ pairs and the length of D2D link is $50 \mathrm{~m}$, with 100 to $120 \mathrm{~m}$ distance of interfering D2D-Txs to the D2D-Rx, the QoS for the D2D-Rx can be guaranteed. While for $10 \mathrm{D} 2 \mathrm{D}$ pairs, it is not possible to attain the D2D-Rx's QoS requirement for a less than $200 \mathrm{~m}$ distance between D2D-Txs and D2D-Rx.

\subsection{2 | Exclusive regions}

Figure 8 illustrates the derived ERs for six different configurations. The D2D-Txs and D2D-Rxs are shown by red and green star symbols, respectively. The blue circle centered at the BS indicates the $\mathrm{ER}_{\mathrm{bd}}$ by which no D2D-Rx is allowed to be inside this circle. The dark green circle (the simulation displays only a part of the circle that is positioned into the considered cell) centered at the $\mathrm{CU}$ shows the $\mathrm{ER}_{\mathrm{dc}}$ in which no D2D-Tx can be located inside the circle. The red circles centered with D2D-Rxs show the $\mathrm{ER}_{\mathrm{dd}}$ for each D2D-Rx in which D2D-Txs of other pairs are not allowed to be located inside the circles. In the configuration in Figure 8A, we set $\gamma_{\text {min }}^{c}=10 \mathrm{~dB}, \gamma_{\text {min }}^{d}=5 \mathrm{~dB}, d_{i}^{b c}=300 \mathrm{~m}$, and $d_{k k}^{d d} \leq$ $50 \mathrm{~m}$. As indicated, with this configuration it is possible to have at maximum $14 \mathrm{D} 2 \mathrm{D}$ pairs admissible for sharing the same spectrum with the CU. We use the configuration in Figure $8 \mathrm{~A}$ as the basis and investigate the impact of the above mentioned parameters in other configurations. In Figure 8B, $\gamma_{\min }^{c}=5 \mathrm{~dB}$, the result shows that it would be possible to share spectrum of the $\mathrm{CU}$ at maximum with $15 \mathrm{D} 2 \mathrm{D}$ pairs, which shows very low improvement compared with the configuration in Figure 8A. In Figure 8C, $\gamma_{\min }^{d}=0 \mathrm{~dB}$, due to the fact that we need to guarantee the QoS target for D2D-Rxs, the simulation result shows a great improvement in the number of admissible D2D pairs. In fact, $\gamma_{\min }^{d}$ is a key factor determining the number of concurrent transmitting D2D pair sharing the spectrum of a CU. Note that with high-QoS requirement for CUs and D2D pairs, the sizes of the ERs will be larger and can limit the number of concurrent D2D pairs in a cell. In Figure 8D and Figure 8E, we consider the impact of the position of the $\mathrm{CU}$ on the number of admissible D2D pairs and the ERs establishment. In cases where the $\mathrm{CU}$ is near the cell border (Figure 8D), the maximum number of D2D pairs is restricted to 13, and when it is closer to the BS (Figure 8E), it is possible to support 17 admissible D2D pairs. In Figure 8F, $d_{k k}^{d d}=30 \mathrm{~m}$, the result shows 25 admissible D2D pairs for spectrum sharing with the CU. Indeed, distance between pairs of D2D links have a great impact on the number of admissible D2D pairs.

\section{2 | QARA performance in a loaded cell}

Up to this point, we have considered the ERs establishment and discussed how to find admissible D2D pairs for each active CU. Now, we investigate performance of QARA algorithm in a loaded network consisting of 8 CUs and 16 D2D pairs.

\subsection{1 | Average throughput}

Figure 9 illustrates cumulative distribution function (CDF) of the average throughput of active CUs using QARA, the Hungarian, R-ICA, and random algorithms. As the HA is an optimal solution for paring only one D2D pair to each CU, and due to the fact that increasing the number of D2D pairs sharing the same spectrum resources with a $\mathrm{CU}$, increases the interference to the $\mathrm{CU}$, the interference to each active $\mathrm{CU}$ is less than other algorithms. Hence, the HA outperforms the other algorithms in average throughput of CUs. QARA can assign more than one D2D pair for resource sharing with each $\mathrm{CU}$, but as it limits interference to sharing CUs, it outperforms R-ICA and random algorithms. It is worth to mention that depending on location of CUs, some of them are eligible to share their resources with more pairs than others while some cannot share at all. 


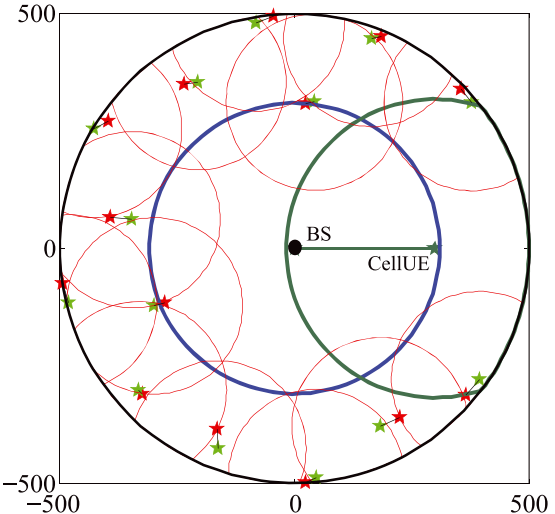

(A)

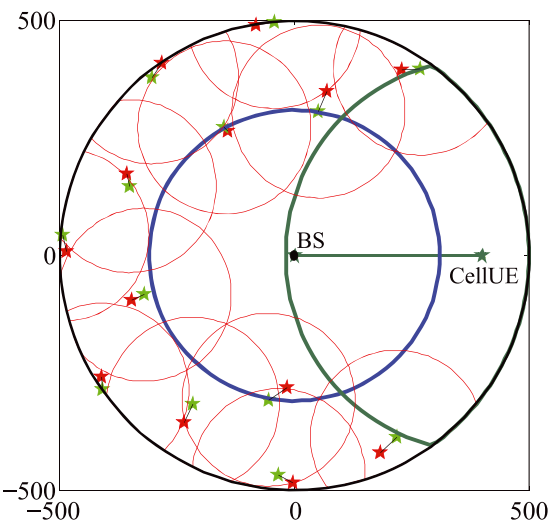

(D)

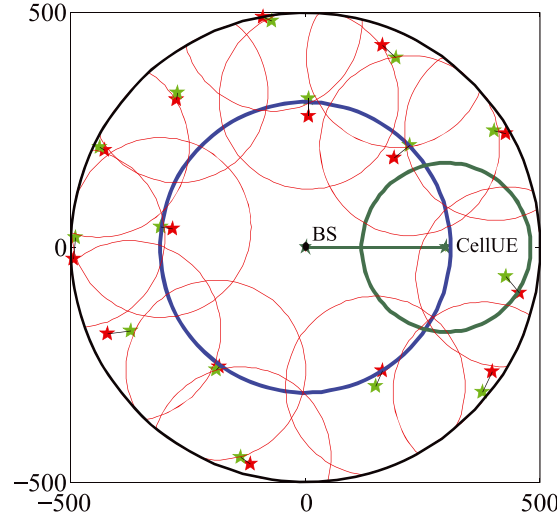

(B)

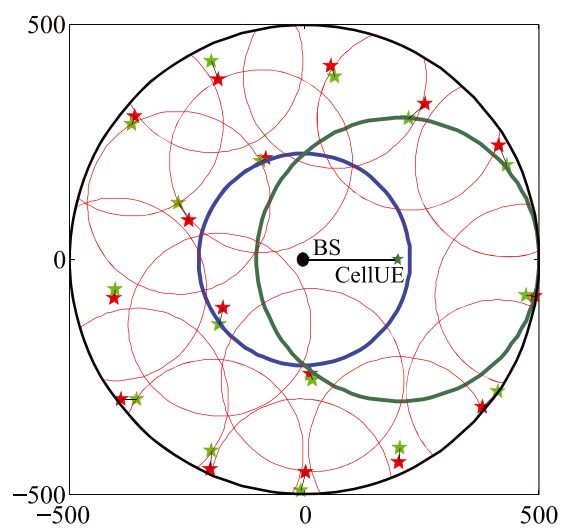

(E)

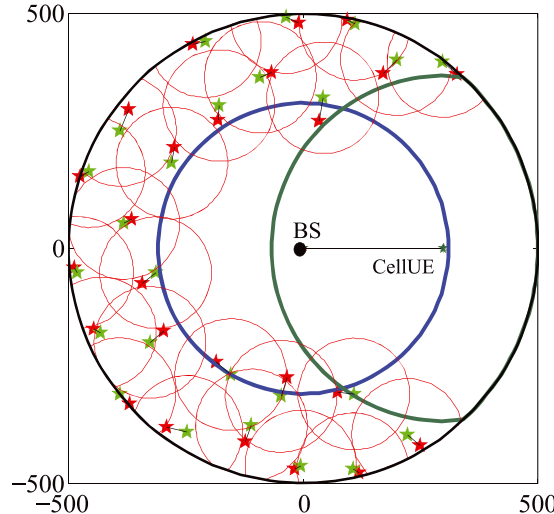

(C)

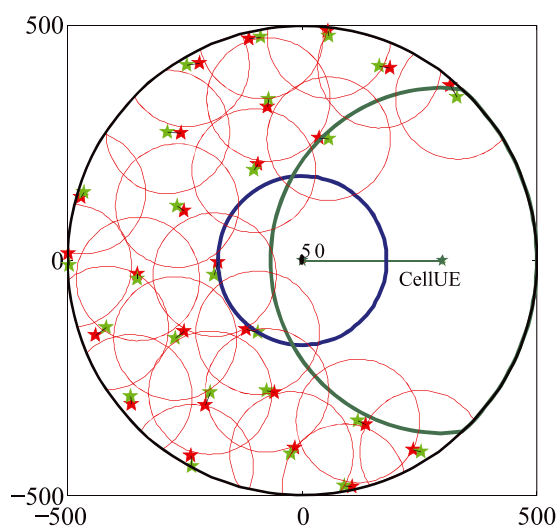

(F)

FIGURE 8 Impact of distance and QoS parameters on ERs establishment and the number of admissible D2D pairs. The parameters used for simulations are in $\mathrm{A}, \gamma_{\min }^{c}=10 \mathrm{~dB}, \gamma_{\min }^{d}=5 \mathrm{~dB}, d_{i}^{b c}=300 \mathrm{~m}, d_{k k}^{d d}=50 \mathrm{~m} . \mathrm{B}, \gamma_{\min }^{c}=5 \mathrm{~dB} . \mathrm{C}, \gamma_{\min }^{d}=0 \mathrm{~dB} . \mathrm{D}, d_{i}^{b c}=400 \mathrm{~m} . \mathrm{E}, d_{i}^{b c}=200 \mathrm{~m} . \mathrm{F}$, $d_{k k}^{d d}=30 \mathrm{~m}$

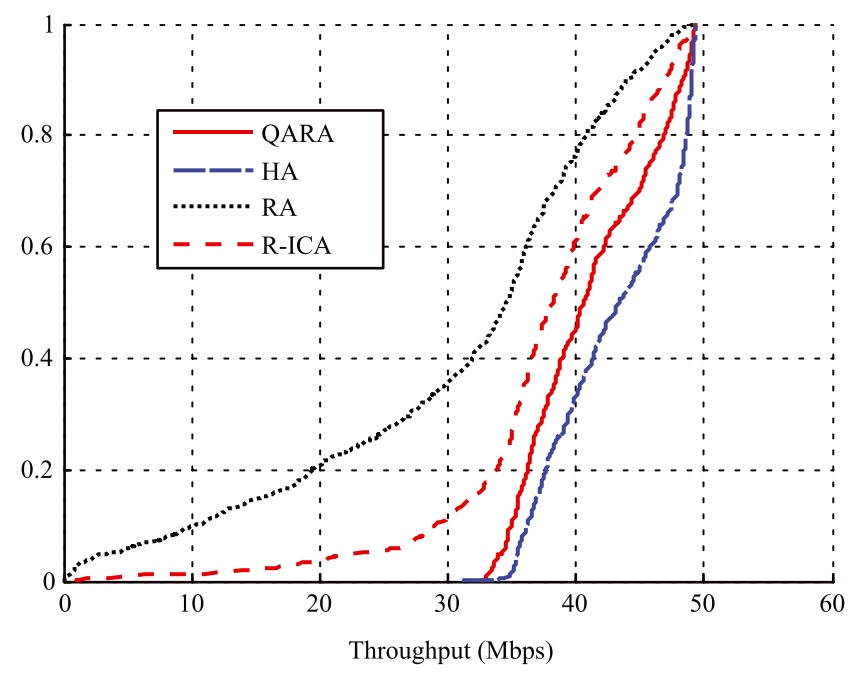

FIGURE 9 CDF of active CU throughput for 8 CUs and 16 D2D pairs

Figure 10 illustrates the CDF of average throughput of the sharing D2D pairs. It can be seen that QARA provides higher throughput than other algorithms. Since QARA takes

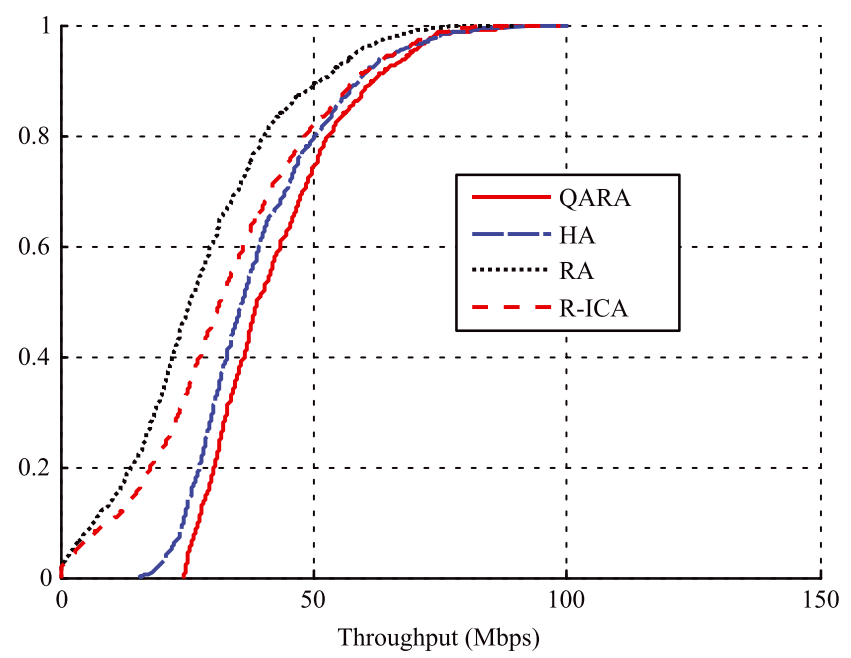

FIGURE 10 CDF of D2D pairs throughput for 8 CUs and 16 D2D pairs

interference into account when choosing D2D pairs for resource sharing, it outperforms other algorithms in term of the average throughput of D2D pairs. 


\subsection{2 | Resource-sharing group measures}

Figure 11 represents the CDF of the size of RSGs when each $\mathrm{CU}$ is paired up with D2D pairs in an independent RSG to communicate on shared spectrum resources. Since R-ICA aims at maximizing network throughput, the sizes of performed groups are bigger than QARA algorithm. While the RA chooses CUs and D2D pairs to be paired up in groups randomly, the HA assigns at most 1 D2D pair for resource sharing with each CU. Note that the QoS targets are assumed to be 10 and $5 \mathrm{~dB}$ for CUs and D2D pairs, respectively. As discussed in Figure 8, with lower QoS requirement values for CUs and D2D pairs, QARA algorithm will perform RSGs with larger size.

Figure 12 outlines the CDF of throughput of RSGs. As discussed earlier, each RSG consist of one active CU and one or several D2D pairs that are selected to communicate on shared spectrum. It can be seen that QARA and the R-ICA algorithms have $10 \%$ and $15 \%$ probability, respectively, of

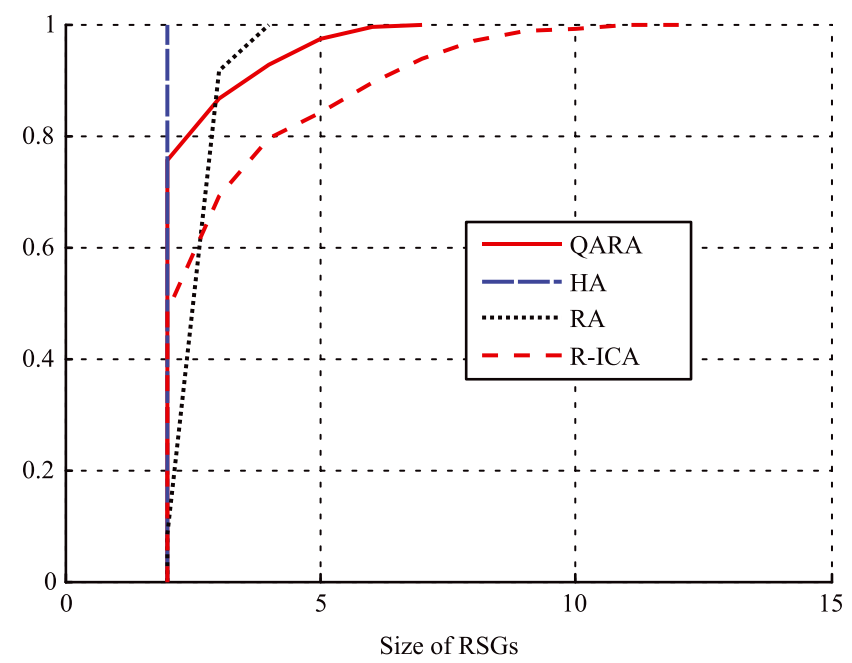

FIGURE 11 CDF of RSGs size performed using different algorithms

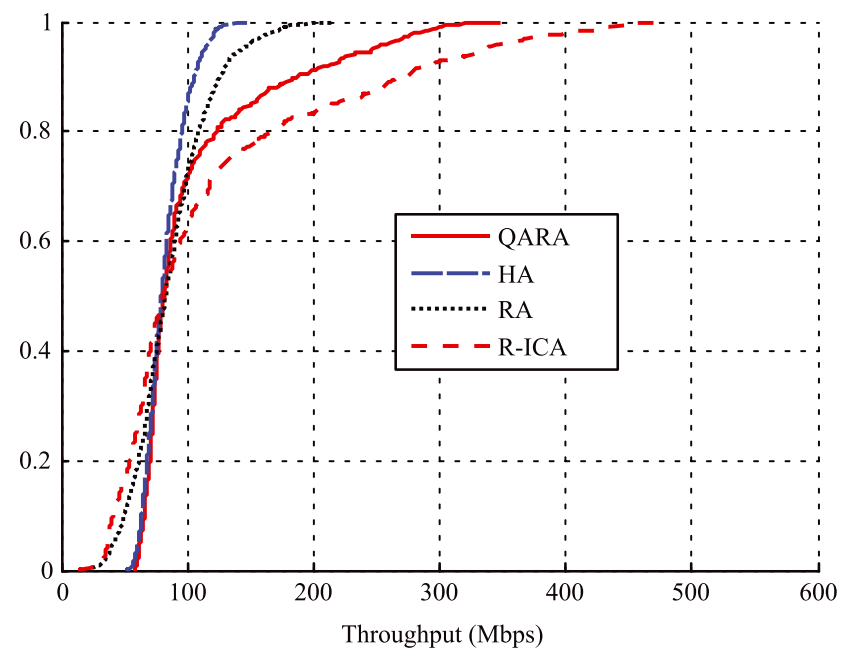

FIGURE 12 CDF of RSG throughput forming RSGs with achievable throughput above $200 \mathrm{Mbps}$. This is due to the fact that R-ICA performs RSGs to maximize network throughput whereas QARA algorithm takes QoS into account in addition to network throughput. On the other hand, probability of providing throughput above $200 \mathrm{Mbps}$ using the HA is negligible, as the HA forms RSGs with only one D2D pair.

\subsection{3 | Total cell throughput}

Figure 13 shows a comparison of total throughput of the cell achieved by the considered algorithms when number of candidate D2D pairs increases from 2 to 16 in a cell with 8 CUs. When the number of D2D pairs is lower than or equal to the number of CUs, the cell throughput achieved by all the algorithms are very close together, but by increasing in number of D2D pairs, R-ICA outperforms other algorithms. QARA provides results comparable to those of the HA for fewer candidate D2D pairs and follows R-ICA with growing number of candidate D2D pairs.

\subsection{4 $\mid$ D2D assignment ratio}

Figure 14 demonstrates D2D assignment ratio when number of $\mathrm{D} 2 \mathrm{D}$ pairs increases from 2 to 16 . D2D assignment ratio is defined as the ratio of number of admitted D2D pairs for

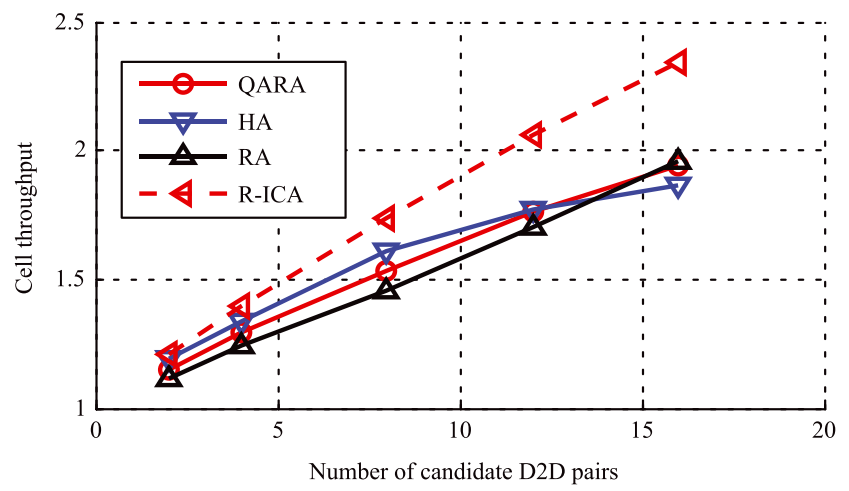

FIGURE 13 Total cell throughput

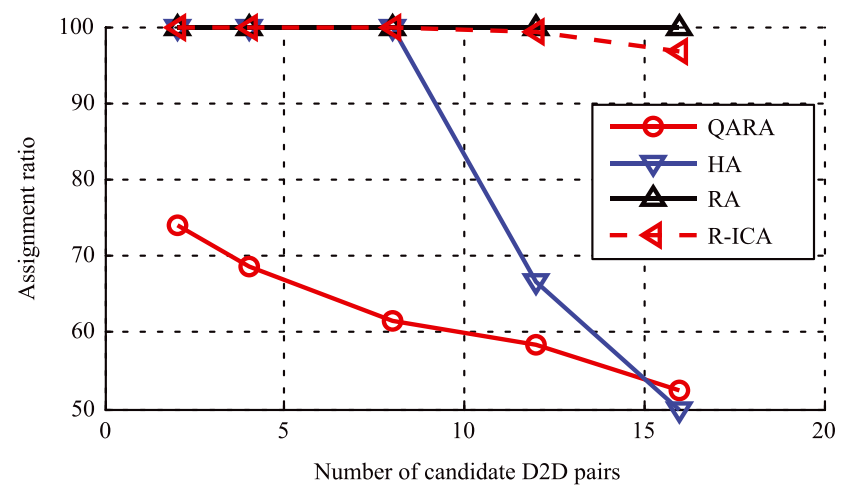

FIGURE 14 D2D assignment ratio 
resource sharing to the total number of D2D pairs in the cell. It represents the efficiency of the resource allocation algorithm. It can be seen that by using QARA algorithm, due to QoS violation, some of $\mathrm{D} 2 \mathrm{D}$ pairs will not be admitted for resource sharing with any one of the active CUs. The HA allocates resources to all D2D pairs when number of D2D pairs are less than or equal to CUs, while R-ICA allocates spectrum resources to almost all of D2D pairs to maximize the overall cell throughput.

\subsection{5 | Outage probability}

Figure 15 indicates the maximum outage probability of active CUs when the QoS requirement for CUs is $10 \mathrm{~dB}$. One can see that QARA algorithm can effectively maintain the outage probability of active CUs less than $10 \%$. The R-ICA provides the highest level of cell throughput but due to the fact that it does not consider CUs' QoS requirement, the CUs suffer from severe interference. Hence, the outage probability of active CUs is very high, especially when number of sharing D2D pairs increases. Moreover, RA can provide a reasonable throughput, but due to the fact that the selection of D2D pairs for resource sharing is totally random, there would be a high probability of choosing not proper D2D pairs for resource sharing with a CU. This may cause significant amount of interference to the active $\mathrm{CU}$ and high outage probability.

Figure 16 demonstrates the maximum outage probability of D2D pairs sharing the same spectrum resource and are chosen to be in a same RSG. Similar to previous case, QARA algorithm, efficiently keeps the outage probability of D2D pairs less than $10 \%$. As discussed earlier, the average size of RSGs using R-ICA are bigger than QARA and the HA; thus, the number of $\mathrm{D} 2 \mathrm{D}$ pairs communicate on the same spec-

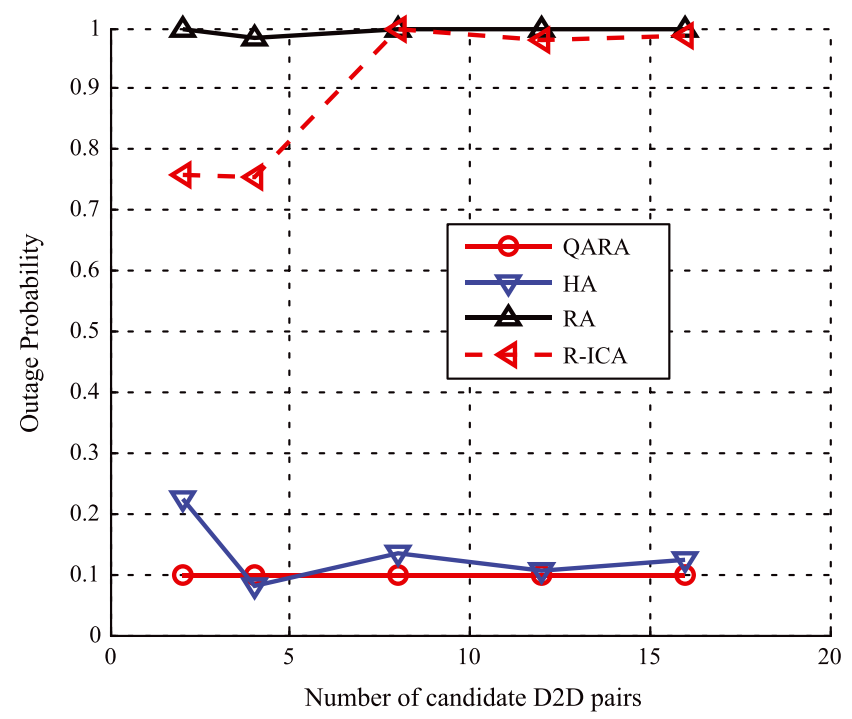

FIGURE 15 Outage probability of active CUs trum are higher than these algorithms. Indeed, interference between D2D pairs is higher, and consequently, the outage probability using R-ICA is relatively higher than in those algorithms.

\subsubsection{Complexity}

Figure 17 shows the computational complexity (mean running time) of the considered algorithms when there are $8 \mathrm{CUs}$ and number of D2D pairs increasing from 2 to 16 . Vertical axis shows decimal logarithm of average running time scaled by 10 . The complexity of the HA and R-ICA are discussed in Han et $\mathrm{al}^{31}$ and $\mathrm{Xu}$ et $\mathrm{al}^{21}$, respectively. While the upper bound complexity of QARA in the worst case is $O\left(N M^{3}\right)$, where $\mathrm{N}$ and $\mathrm{M}$ represent the number of active CUs and candidate $\mathrm{D} 2 \mathrm{D}$ pairs, respectively. The proposed algorithm has the lowest complexity in comparison to other algorithms and also very low increase in its complexity by increasing in the number of D2D pairs. It can be clearly seen that R-ICA running time grows exponentially with the number of considered D2D

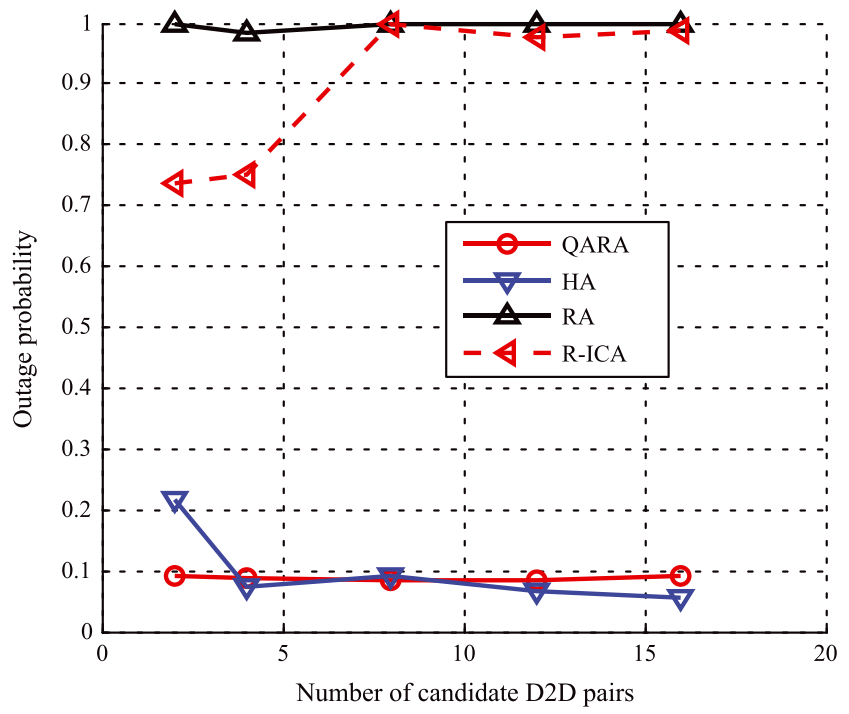

FIGURE 16 Outage probability of sharing D2D pairs

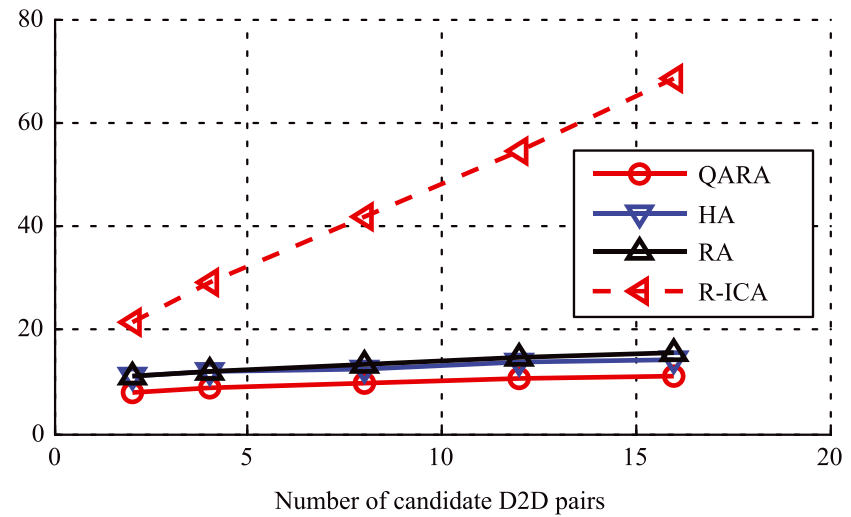

FIGURE 17 Complexity of the algorithms 
pairs. Complexity of R-ICA is about 10 times higher than other algorithms for 2 D2D pairs and approximately 1 million times higher for $16 \mathrm{D} 2 \mathrm{D}$ pairs. It is due to the fact that R-ICA perform exhaustive search for optimal D2D pairs for spectrum resource allocation.

Summing up the results discussions, R-ICA is an efficient algorithm that can maximize the overall cell throughput but it suffers from two main drawbacks. First, computational complexity of the algorithm is very high and exponentially increases with the number of D2D pairs. Second, R-ICA does not consider the QoS requirements for CUs and D2D pairs. Hence, in scenarios that QoS and/or computational complexity are important factor, R-ICA is not an applicable algorithm. Traditionally, the HA has been proposed as an optimal solution for pairing algorithm that exploit exhaustive search for paring of single D2D pair with each CU. However, it is not a suitable solution for multi-D2D pair resource-sharing scenarios, specifically when the number of D2D pairs is larger than the number of active CUs. QARA can be seen as an efficient resource allocation framework for D2D communications. The first two steps of algorithm can be used for one CU-1 D2D pair resource allocation that provides near optimal performance with low computational complexity. QARA as a whole algorithm is used for one CU-multiple D2D pairs resource allocation that provides high-performance level with relatively low complexity. Furthermore, in comparison to the other considered algorithms, QARA algorithm is a more efficient approach to avoid or reduce interference to CUs and D2D pairs. In fact, QARA algorithm can efficiently guarantee the required QoS for CUs and D2D-Rxs. Besides, QARA algorithm enables us, based on applications requirements, to define different QoS target values for cellular and D2D communications, leading to different network performance and RSGs formation. The flexibility and advantages of QARA algorithm makes it an efficient method for cooperative content delivery in 5G networks, resulting particularly attractive in loaded cases.

\section{6 | CONCLUSIONS AND FURTHER DISCUSSIONS}

In this paper, we have investigated resource allocation for multi pairs D2D communications underlaying cellular network in a single cell. We formulated an optimization problem to maximize network throughput while guaranteeing the QoS requirements for CUs and D2D pairs. We devised a four-step QARA algorithm to address the problem. The QARA algorithm exploits a distance-based admission control method to drive set of RSGs that show the D2D pairs that are admitted to communicate by sharing spectrum resource of each particular CU. We evaluated performance of the QARA algorithm and compared with R-ICA, the HA, and RA in terms of network throughput, outage probability, and computational complexity. The analysis of the numerical results demonstrates that with small numbers of D2D pair candidates, QARA provides near optimal network throughput, similar to the HA. In a loaded network, when the numbers of D2D pair candidates are higher than CUs, QARA outperforms the HA and follows R-ICA that provides maximum network throughput. While there is high probability of outage for CUs and D2D pairs with using R-ICA for resource allocation, QARA algorithm can efficiently guarantee QoS for both cellular and D2D communications and maintain the outage probability of active CUs and D2D pairs less than $10 \%$. Furthermore, QARA performs resource allocation with lower computational complexity than R-ICA. In the future, we will extend optimal resource allocation for multi-D2D pair communications in multi cells.

\section{REFERENCES}

1. Boccardi F, Heath R, Lozano A, Marzetta T, Popovski P. Five disruptive technology directions for 5G. IEEE Commun Mag. 2014;52(2):74-80.

2. Bangerter B, Talwar S, Arefi R, Stewart K. Networks and devices for the 5G era. IEEE Commun Mag. 2014;52(2):90-96.

3. Andrews J, Buzzi S, Choi W, et al. What will $5 \mathrm{G}$ be? IEEE J Sel Areas Commun. 2014;32(6):1065-1082.

4. Bagheri H, Katz M, Fitzek F, Lucani D, Pedersen M. D2D-based mobile clouds for energy- and spectral-efficient content distribution. In: Mumtaz S, Rodriguez J, eds. Smart Device to Smart Device Communication. Oxford: Springer; 2014; 237-280.

5. Fodor G, Dahlman E, Mildh G, et al. Design aspects of network assisted device-to-device communications. IEEE Commun Mag. 2012;50(3):170-177.

6. Doppler K, Rinne M, Wijting C, Ribeiro C, Hugl K. Device-to-device communication as an underlay to LTE-advanced networks. IEEE Commun Mag. 2009;47(12):42-49.

7. Huang Y, Nasir AA, Durrani S, Zhou X. Mode selection, resource allocation, and power control for D2D-enabled two-tier cellular network. IEEE Trans Commun. 2016;64(8):3534-3547.

8. Hakola S, Chen T, Lehtomaki J, Koskela T. Device-to-device (d2d) communication in cellular network - performance analysis of optimum and practical communication mode selection. Paper presented at: IEEE Wireless Communications and Networking Conference (WCNC); 2010; Sydney.

9. Xu X, Wang H, Feng H, Xing C. Analysis of device-to-device communications with exclusion regions underlaying $5 \mathrm{G}$ networks. Trans Emerging Tel Tech. 2015;26(1):93-101.

10. Lin M, Ouyang J, Zhu WP. Joint beamforming and power control for device-to-device communications underlaying cellular networks. IEEE J Sel Areas Commun. 2016;34(1):138-150.

11. Lee N, Lin X, Andrews JG, Heath RW. Power control for D2D underlaid cellular networks: modeling, algorithms, and analysis. IEEE J Sel Areas Commun. 2015;33(1):1-13.

12. Wang $\mathrm{H}, \mathrm{Chu} \mathrm{X}$. Distance-constrained resource-sharing criteria for device-to-device communications underlaying cellular networks. Electron Lett. 2012;48(9):528-530.

13. Min H, Lee J, Park S, Hong D. Capacity enhancement using an interference limited area for device-to-device uplink underlaying 
cellular networks. IEEE Trans Wireless Commun. 2011;10(12): 3995-4000.

14. Golrezaei N, Molisch AF, Dimakis AG, Caire G. Femtocaching and device-to-device collaboration: a new architecture for wireless video distribution. IEEE Commun Mag. 2013;51(4): 142-149.

15. Golrezaei N, Mansourifard P, Molisch A, Dimakis A. Base-station assisted device-to-device communications for high-throughput wireless video networks. IEEE Trans Wireless Commun. 2014;13(7):3665-3676.

16. Bagheri H, Miranda Bonomi FA, Katz M. Spectral efficiency and throughput enhancement by full-duplex D2D communication in mobile clouds. Paper presented at: Proceedings of the 21th European Wireless Conference; 2015; Budapest.

17. Zulhasnine M, Huang C, Srinivasan A. Efficient resource allocation for device-to-device communication underlaying LTE network. Paper presented at: 2010 IEEE 6th International Conference on Wireless and Mobile Computing, Networking and Communications (WIMOB); 2010; Niagara Falls.

18. Kaufman B, Lilleberg J, Aazhang B. Spectrum sharing scheme between cellular users and ad-hoc device-to-device users. IEEE Trans Wireless Commun. 2013;12(3):1038-1049.

19. Ni M, Pan J, Cai L. Geometrical-based throughput analysis of device-to-device communications in a sector-partitioned cell. IEEE Trans Wireless Commun. 2015;14(4):2232-2244.

20. Bagheri H, Katz M. A resource allocation mechanism for enhancing spectral efficiency and throughput of multi-link D2D communications. Paper presented at: IEEE 25th Annual International Symposium on Personal, Indoor, and Mobile Radio Communication (PIMRC); 2014; Washington.

21. Xu C, Song L, Han Z, et al. Efficiency resource allocation for device-to-device underlay communication systems: a reverse iterative combinatorial auction based approach. IEEE J Sel Areas Commun. 2013;31(9):348-358.

22. Li P, Guo S, Stojmenovic I. A truthful double auction for device-to-device communications in cellular networks. IEEE J Sel Areas Commun. 2016;34(1):71-81.

23. Kebriaei H, Maham B, Niyato D. Double-sided bandwidth-auction game for cognitive device-to-device communication in cellular networks. IEEE Trans Veh Technol. 2016;65(9):7476-7487.

24. Song L, Niyato D, Han Z, Hossain E. Game-theoretic resource allocation methods for device-to-device communication. IEEE Wireless Commun. 2014;21(3):136-144.

25. Li Y, Jin D, Yuan J, Han Z. Coalitional games for resource allocation in the device-to-device uplink underlaying cellular networks. IEEE Trans Wireless Commun. 2014;13(7):3965-3977.

26. Feng D, Lu L, Yuan-Wu Y, Li G, Feng G, Li S. Device-to-device communications underlaying cellular networks. IEEE Trans Commun. 2013;61(8):3541-3551.

27. Phunchongharn P, Hossain E, Kim D. Resource allocation for device-to-device communications underlaying LTE-advanced networks. IEEE Wireless Commun. 2013;20(4):91-100.

28. Belleschi M, Fodor G, Abrardo A. Performance analysis of a distributed resource allocation scheme for D2D communications. Paper presented at: IEEE GLOBECOM Workshops (GC Wkshps); 2011; Houston.

29. Yu G, Xu L, Feng D, Yin R, Li G, Jiang Y. Joint mode selection and resource allocation for device-to-device communications. IEEE Trans Commun. 2014;62(11):3814-3824.

30. Jung M, Hwang K, Choi S. Joint mode selection and power allocation scheme for power-efficient device-to-device (d2d) communication. Paper presented at: IEEE 75th Vehicular Technology Conference (VTC Spring); 2012; Yokohama.
31. Han J, Cui Q, Yang C, Tao X. Bipartite matching approach to optimal resource allocation in device to device underlaying cellular network. Electron Lett. 2014;50(3):212-214.

32. Wang L, Tang H, Wu H, Stuber GL. Resource allocation for D2D communications underlay in Rayleigh fading channels. IEEE Trans Veh Technol. 2016;PP(99):1-1.

How to cite this article: Bagheri H, Miranda Bonomi FA, Katz M. A low-complexity QoS aware resource allocation algorithm for multi pair deviceto-device (D2D) communications. Trans Emerging Tel Tech. 2017;28:e3186. https://doi.org/10.1002/ett.3186

\section{APPENDIX}

Proof. Proof of Lemma 1

$$
P_{i}^{\text {Out }, c}=\operatorname{Pr}\left(\frac{Z_{i}^{b c}}{\sum_{d_{k} \in G_{i}} Z_{k i}^{d c}+P_{\mathrm{n}}} \leq \gamma_{\min }^{c}\right) .
$$

Taking the expected value over every interfering D2D signal $Z_{k i}^{d c}$

$$
\begin{aligned}
P_{i}^{\text {Out }, c} & =\underset{Z_{k i}^{d c}}{\mathbb{E}}\left[\operatorname{Pr}\left(\frac{Z_{i}^{b c}}{\sum_{d_{k} \in G_{i}} Z_{k i}^{d c}+P_{\mathrm{n}}} \leq \gamma_{\text {min }}^{c}\right)\right] \\
& =\underset{Z_{k i}^{d c}}{\mathbb{E}}\left[\operatorname{Pr}\left(Z_{i}^{b c} \leq \gamma_{\min }^{c}\left(\sum_{d_{k} \in G_{i}} Z_{k i}^{d c}+P_{\mathrm{n}}\right)\right)\right] .
\end{aligned}
$$

As $Z_{i}^{b c}$ is exponentially-distributed with mean $\bar{Z}_{i}^{b c}$

$$
\begin{aligned}
& P_{i}^{\text {Out }, c}=\underset{Z_{k i}^{d c}}{\mathbb{E}}\left[1-e^{-\frac{\gamma_{\min }^{c}\left(\sum_{d_{k} \in G_{i}} Z_{k i}^{d c}+P_{\mathrm{n}}\right)}{\bar{Z}_{i}^{b c}}}\right], \\
& =1-e^{-\frac{\gamma_{\min }^{c} P_{n}}{Z_{i}^{b c}}} \underset{Z_{k i}^{d c}}{\mathbb{E}}\left(e^{-\frac{\gamma_{\min }^{c} \sum_{k} \in G_{i} Z_{k i}^{d c}}{Z_{i}^{b c}}}\right), \\
& =1-e^{-\frac{\gamma_{\min }^{c} P_{\mathrm{n}}}{\bar{z}_{i}^{\overline{b_{i}}}}} \prod_{d_{k} \in G_{i}} \underset{Z_{k i}^{d c}}{\mathbb{E}}\left(e^{-\frac{\gamma_{\min }^{c} Z_{k i}^{d c}}{\bar{z}_{i}^{c c}}}\right) .
\end{aligned}
$$

Now, we calculate the expected value in Al for a generic exponentially distributed random variable $X$ with mean $\bar{x}$. 


$$
\begin{aligned}
\underset{X}{\mathbb{E}}\left(e^{-\frac{\gamma_{\min }^{c} x}{\bar{z}_{i}^{b c}}}\right) & =\int_{0}^{\infty} e^{-\frac{\gamma_{\min }^{c} x}{\bar{Z}_{i}^{b c}}} \frac{1}{\bar{x}} e^{-\frac{x}{\bar{x}}} \mathrm{~d} x \\
& =\frac{1}{\bar{x}} \int_{0}^{\infty} e^{-\frac{\gamma_{\min }^{c} \bar{x}+\bar{z}_{i}^{b c}}{\bar{x} \bar{z}_{i}^{b c}} x} \mathrm{~d} x \\
& =\frac{\bar{Z}_{i}^{b c}}{\gamma_{\min }^{c} \bar{x}+\bar{Z}_{i}^{b c}}, \\
& =\left(1+\frac{\gamma_{\min }^{c} \bar{x}}{\bar{Z}_{i}^{b c}}\right)^{-1}
\end{aligned}
$$

As every $Z_{k i}^{d c}$ is exponentially-distributed with mean $\bar{Z}_{k i}^{d c}$, applying Equation A2 to Equation A1, we obtain

$$
P_{i}^{\text {Out }, c}=1-e^{-\frac{\gamma_{\min }^{c} P_{\mathrm{n}}}{\overline{\bar{Z}}_{i}^{b c}}} \prod_{d_{k} \in G_{i}}\left(1+\frac{\gamma_{\min }^{c} \bar{Z}_{k i}^{d c}}{\bar{Z}_{i}^{b c}}\right)^{-1}
$$

